\title{
USING COMPETENCY QUESTIONS TO EVALUATE AN AGENT-BASED MODEL FOR VIRTUAL ENTERPRISES
}

\author{
Sobah Abbas Petersen \\ Dept. of Computer and Information Science, Norwegian University of Science and \\ Technology, Trondheim, NORWAY \\ Email: sap@idi.ntnu.no
}

\begin{abstract}
This paper presents an agent-based model to support the formation of Virtual Enterprises, where the partners of a Virtual Enterprise are represented by software agents. The model describes the main concepts: goals, activities, roles, the requirements for the roles, the agents' skill and competencies and the relationships among these different concepts. We believe that in order to be able to provide the necessary support, the model must be able to provide answers to questions such as what are the skills required to perform a particular activity, or what goal(s) does a partner contribute to? We evaluate our model by applying such questions derived from literature and a series of case studies.
\end{abstract}

\section{INTRODUCTION}

A Virtual Enterprise (VE) is a scenario where a number of units (or partners), who may be human beings, organisations or software agents, collaborate to achieve the goals of the VE. In this paper, we consider an agent-based model of a VE, where the partners in a VE are represented by software agents (or agents). Our model is based on the ideas of Enterprise Modelling where the model describes the main concepts: goals, activities, roles, the requirements for the roles, the agents' skill and competencies and the relationships among these different concepts. We focus on the formation phase of the lifecycle of a VE, where the model supports this process by providing information about the roles of the $\mathrm{VE}$, the requirements for the roles and the relationships of the agents to the rest of the entities in the model. However, the role of models is no longer just that of an information repository; rather models are now required to support decision-making and to answer queries about what it represents, [6].

Although there are a number of ways of modelling an enterprise, (see [14] for an overview), there is no clear way to evaluate an enterprise model, [4]. Fox et. al. identified the need for the evaluation of models for a number of reasons such as the need to identify when the modelling is completed and the model should be made operational or to judge the correctness of the representation. The notion of 
competency questions was introduced, where the competency of a model is its ability to answer questions or its deductive capability. We have chosen the idea of competency questions to evaluate our agent-based model of a VE. Our aim is to design a set of questions to evaluate our model, which can also be used as an evaluation framework for agent-based models to support the formation of VEs.

In this paper, we describe how our model is evaluated using a set of competency questions, derived from literature and a series of case studies. The remainder of this paper is organised as follows: Section 2 describes the notion of competency questions, Section 3 gives an overview of our model, Section 4 gives a brief description of the industrial case studies, Section 5 provides the list of competency questions, Section 6 describes the evaluation conducted by querying the model, Section 7 discusses the results of the evaluation and Section 8 concludes this paper.

\section{COMPETENCY QUESTIONS}

A model's ability to answer a set of questions gives an indication of the competency of a model or how well it supports problem solving. Competency questions represent a set of tasks that arise in enterprise engineering and the requirements on the enterprise model that is required to represent the tasks and their solutions, [6] and [7]. These requirements are formulated as a set of questions that the model is required to answer.

One of the advantages in using competency questions is that they can be informal questions that can be answered informally or they can be specified formally to provide a more precise evaluation of the model. The idea of competency questions was applied in [5], where they described a set of questions that were answered by an enterprise model described in First Order logic, using deductive reasoning. Their model focussed on the operational aspects of an enterprise and answered questions with respect to planning and scheduling, temporal projection and execution and monitoring of events.

There are several ways of evaluating models; for example, agent-based systems can be evaluated by formal methods such as model checking, (e.g. [16]), where a system is verified against its specifications. A framework for evaluating the quality of interactive and conceptual models was presented in [9]. In [10], a framework for comparing and evalutaing information models was presented. Other approaches for evaluation of models include case studies, [17]. Although there are approaches to evaluate a conceptual model and agent-based system against their specifications, there is no established means of evaluating Enterprise Models. We have used case studies to evaluate our approach in general and to strengthen our set of competency questions.

\section{MODEL OF A VIRTUAL ENTERPRISE}

An agent-based model for a VE was proposed in [12], where the entities in a VE and their relationships were described; see Figure 1. The model consists of the goals of the VE, the activities that must be performed to achieve the goals, the roles to perform the activities and the requirements for the roles. Agents can be selected to 
fill the roles, where the capabilities of an agent must meet the requirements for the roles. The entities in the model are defined in terms of a set of attributes. The main inspiration for our model is TOVE (TOronto Virtual Enterprise), [3], where a formal description of an enterprise is given.

In our model, we use predicate calculus to describe the entities and their attributes and the relationships between the entities. A set of rules defines how the model can be used and the kinds of operations or queries that can be performed on the model. We believe the model must be described in a way that is understandable for the users. Our model aims to address the formation of VEs in industry and thus our users will be the VE engineers working in industry. While a formal representation of a model will provide easy conversion to a computer-based model, non-logicians do not always understand it. In this paper, we have adopted a semiformal approach to describe the contents of our model, where the descriptions of the entities and their relationships are specifications for a formal model.

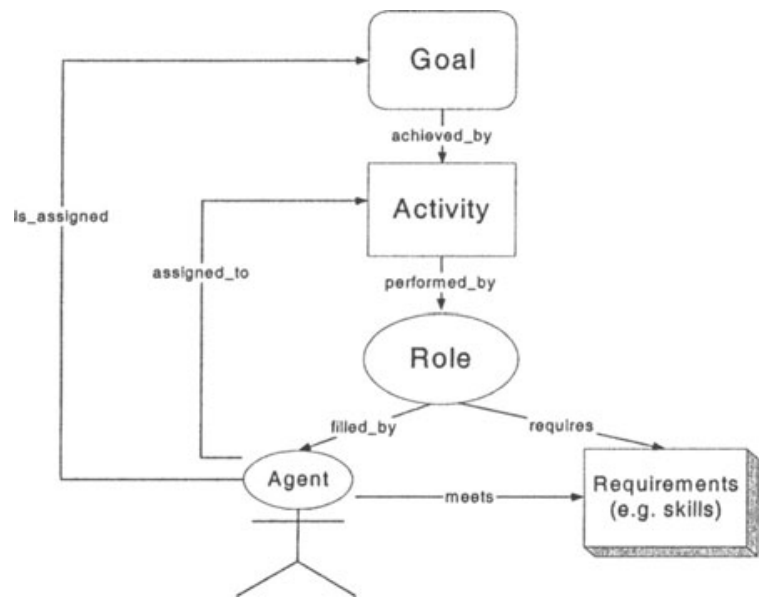

Figure 1: Model of a Virtual Enterprise

A description of the complete model is beyond the scope of this paper. However, we will describe the model briefly by illustrating a few examples of how the entities in the model can be represented. For example, the goal of the VE can be represented as a goal structure, where the main goal can be decomposed into sub-goals. Similarly, the activities are also a structure of activities and sub-activities. Each goal or sub-goal is represented as a set of attributes; the name of the goal, the product area that the VE intends to work in, the delivery date for the product and the total amount of money that the VE can spend to achieve the goal. The goal and the goal structure are represented (in Prolog clauses) as follows:

goal (goal_name, product_area, deadline, max_cost). is_sub_goal (goal1, goal2).

A similar,representation is used to define the activities and its attributes and the activities structure of the VE. The relationships between the entities in the model can be represented as follows: 


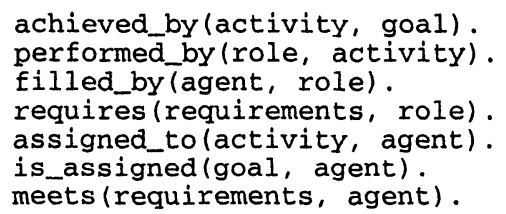

Additional indirect relationships between the entities can be defined by using rules; e.g., the relationship between an agent and an activity can be defined using the following Prolog-like rule:

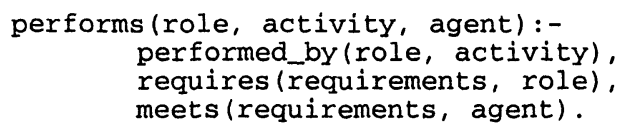

\section{DESCRIPTION OF THE CASES}

We have tried to cover a variety of application domains in our case studies. We primarily looked for situations where a VE was formed with a set of partners that could be either individuals or organisations. Details of the case studies are available from [11] and [13]. The questions that they would like the model to answer were obtained through interviews and discussions. Below is a brief overview of the case studies:

- Company A: Small Consulting Company: An independent private consulting company that offers all aspects of business, financial, economic and social consulting services. It maintains a database of highly qualified consultants in various fields and draws upon these resources to form the teams that work on their projects. These consultants are analogous to the partners of the VE.

- Company B: Student Project Groups: A company that operates in the oil and offshore industry, on a global scale. The particular scenario that we analysed was the selection of several groups of students who will work together as teams during their summer holidays on some projects specified by Company B. Each team, in this case can be considered as a VE.

- Company C: Building Construction Project: Company C is hired by a customer to assist them to evaluate bids in the selection of a contractor for large scale projects in various domains; e.g. in the construction industry for the construction of a hospital. They specialise in the selection of the project team or the partners of the VE that will eventually be formed to deliver to the customer.

\section{FORMULATING A SET OF COMPETENCY QUESTIONS}

The main challenge in using competency questions to evaluate the model is the design of an appropriate set of questions. For our work, the main purpose of the set of competency questions is to check if the model is adequate to support the formation of VEs. We consider 2 crucial questions in organisational design, raised by Bernus et. al., [1], : 
1. How can the task structure of an enterprise be designed to form a coordinated whole?

2. What task allocation to humans (or groups) ensures that the enterprise will act to satisfy its objectives?

We believe that these questions can be used as guidelines in defining a set of detailed questions that can be used to evaluate our model. These questions are rather highlevel and need to be elaborated and expressed in more detail to be able to use them as an evaluation framework for VEs. The notion of "coordination", addressed in question 1, can be viewed from different perspectives, e.g. from an organisational perspective, to manage the products and resources, [2]. We consider coordination from an agent-based perspective, where coordination can be the process in which the agents ensure that a community of individual agents acts in a coherent manner, through joint intentions and plans or by sharing common goals, [8]. We interpret the notion of "objectives", addressed in question 2, as the goals of the VE. Based on these ideas, we can formulate the following questions:

Q1: Do all agents contribute to the goals of the VE?

Q2: Have all goals been assigned agents?

Q3: How well does the agent meet the requirements for the role?

Q4: Is the agent working on any other sub-goals of the VE at the same time?

In addition to the above questions, we have also obtained some questions based on industrial case studies.

Q5: How can we know when VE formation is completed?

Q6: What are the skills required to perform a particular activity?

$Q 7$ : Which activities and goals are affected by the lack of a particular skill?

Q8: Which activities are dependent on each another?

\section{QUERYING THE MODEL}

In this section, we describe how the model can be used to provide answers to the competency questions. The model can be queried using a set of prolog-like rules and we present the pseudo-code for these rules.

\section{Q1: Do all agents contribute to the goals of the VE?}

This question can be asked to determine if all the agents work towards achieving the goals of the VE. If all the agents in the model are assigned a sub-goal of the VE, then they contribute towards the goals of the VE.

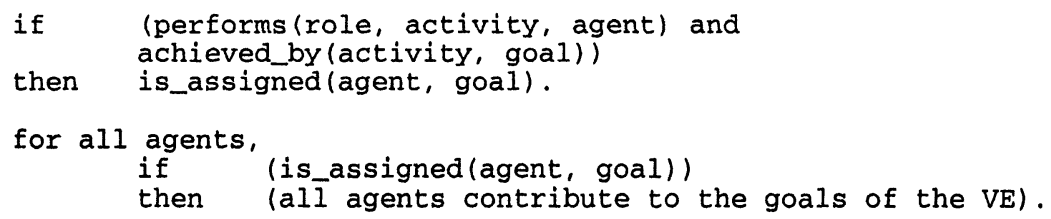

The statement is_assigned(agent, goal) can also be used to identify the subgoal(s) that each agent contributes to. 
Q2: Have all goals been assigned agents?

This question is related to $Q 1$ and help answer if work is done to achieve all the goals in the VE.

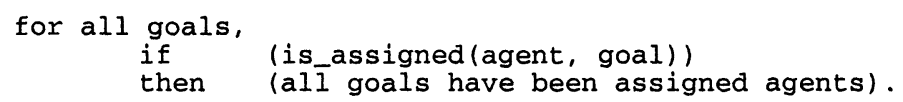

\section{Q3: How well does the agent meet the requirements for the role?}

The requirements for a role are matched against the capabilities of an agent and agents that meet the requirements are selected. The requirements of a VE or the capabilities of an agent are a list of attributes; the capabilities will be structured as the requirements (the requirements reflect the invitation to bid). The requirements or the capabilities may be described as a flat or nested list of attributes. The general rule for matching agents to roles can be described as follows, where the rule match (requirements, capabilities) can be defined as required:

$\begin{array}{ll}\text { if } & \text { (has (agent, capabilities) and } \\ \text { requires (requirements, role) and } \\ \text { match(requirements, capabilities)) } \\ \text { then meets (requirements, agent). }\end{array}$

The matching process in itself is not a simple process; it can involve matching several types of requirements such as skills, availability and price. We consider a simple example where some skills are matched, using the following Prolog-like rule:

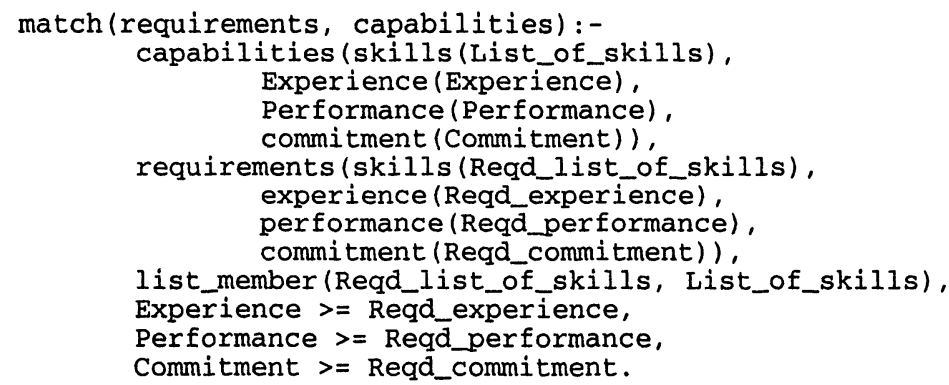

\section{Q4: Is the agent working on any other sub-goals of the VE at the same time?}

It can be important to identify if an agent is working towards more than one subgoal for several reasons such as to identify sub-teams within the VE (assuming that members of a team share a common goal). It could also help identify if an agent performs more than one role simultaneously. In this situation, it might be necessary to reconsider an agent's time allocation and assignment of activities. This question will thus help identify situations that may require negotiation upon an agent's use of time.

For any agent, if the statement is_assigned (agent, goal) is true for more than one value of goal, then the agent is assigned to more than one sub-goal of the VE. The same line of reasoning can be used to identify agents that work towards the same sub-goal of the VE. 


\section{Q5: How can we know when VE formation is completed?}

Since the formation of the VE is one phase in the lifecycle of the VE and the operation of the VE is dependent on the formation of the VE, this is an important question. The VE formation process is completed when agents are assigned to all roles. (We assume that the model of the VE is complete such that all goals are assigned activities and that all activities have roles to perform them.) The following rule can be used to check if all roles have agents assigned to them:

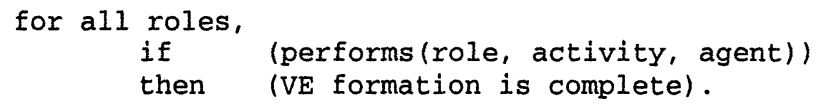

\section{Q6: What are the skills required to perform a particular activity?}

Skills are often the most important requirements for roles, specially for activities that require high specialisation of skills, e.g. the design of a hospital in Company C.

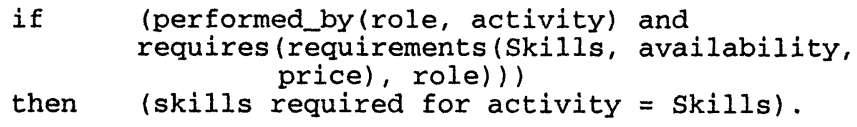

\section{Q7: Which activities and goals are affected by the lack of a particular skill?}

The lack of a particular skill or competence can have significant consequences for a VE, e.g. it can be expressed in terms of cost or time. For any role where the required skill or competence is unavailable, the following rule can be applied to identify the activities and goals that will be affected by it.

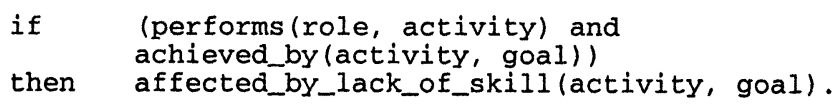

\section{Q8: Which activities are dependent on each another?}

Activities can be dependent on each other due to a number of reasons such as an overlap due to their start and completion dates, an output from one activity is an input to another and the same agent is performing the activities. The dependencies between activity 1 and activity 2 can be defined as follows:

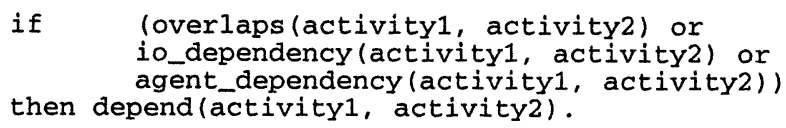

Then, rules for the different kinds of dependencies, e.g. overlap due to time, can be specified as follows:

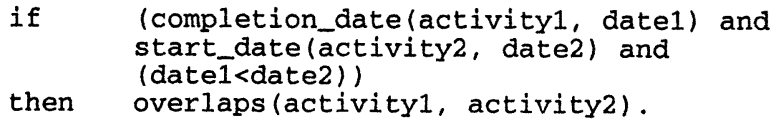




\section{DISCUSSION}

In this model, we have focussed on the entities and the relationships between the entities. Thus, the questions are focussed on this and they help answer queries related to what entity is affected rather than how an entity is affected. For example, if we had a question such as "How are the goals affected by the lack of a particular skill?", then we would need to enhance the model to be able to provide a complete answer to the question.

The competency questions evaluate the competency of a model. However, for a complete evaluation of the model, other characteristics of the model must also be evaluated. In [4], Fox et. al. proposed a set of characteristics to evaluate an enterprise model. These characteristics are generality, competence, efficiency, perspicuity, transformability, extensibility, granularity and scalability. It is interesting to view our model in terms of the other evaluation criteria.

- Generality: although the analysis of the case studies is not discussed in this paper, we believe the model can be used for VEs in different application areas.

- Tranformability and efficiency: since the model can be described in a formal way, it can be easily transformed into a computer-based model that can be used for efficient reasoning.

- Perspicuity: since the model can be described in a semi-formal syntax, the users can understand it.

- Granularity and Scalability: we have focussed on the formation phase of the lifecycle of the VE. However, we believe that the model can be enhanced, by adding appropriate attributes to the entities, to cover the complete lifecycle. We have also identified additional attributes to describe agents' capabilities, e.g. an agent's capability to cooperate.

- Extensibility: the model is designed to cover the generic aspects of a VE, such that it can be extended to include other concepts. One such concept could be the notion of change, e.g. how do we know when a goal has changed? In this paper, we have not addressed issues such as this. However, the model can be extended to include rules to detect when a change occurs, e.g.:

$$
\begin{aligned}
& \text { if } \begin{array}{l}
\text { change_in_goal_attributes (goal) or } \\
\text { change_in_goal_structure(goal)) }
\end{array} \\
& \text { then in_goal(goal). }
\end{aligned}
$$

\section{CONCLUSION}

This paper describes how an agent-based model of a VE can be evaluated using a set of competency questions. The set of questions were formulated based on literature and some industrial case studies. The aim of this paper is to illustrate that our agentbased model for the formation of a VE can be evaluated using a set of competency questions. While the model is able to answer the set of questions adequately, the main challenge in this approach is the design of the set of questions themselves. The 
result of the evaluation of the model is dependent on the relevancy of the questions it is asked.

The set of questions we have used represent the types of questions that a model is required to answer. In the future, we plan to enhance this set by extensive review of the related literature, for example [15], and case studies in order to present a framework for evaluating agent-based models to support VEs.

\section{Acknowledgements}

This work is partially supported by the Norwegian Research Foundation in the framework of the Information and Communication Technology (IKT-2010) program and the ADIS project. The author would like to thank Monica Divitini for her valuable comments and feedback and the people who provided the inputs for the case studies.

\section{REFERENCES}

[1]. Bernus,P., Nemes,L., Organisational Design: Dynamically Creating and Sustaining Integrated Virtual Enterprises, Proc. of IFAC World Congress, Han-Fu Chen, Dia-Zhan Cheng and JiFeng Zhang (Eds) Vol-A, London: Elsevier, 1999, pp.189-194.

[2]. Bernus,P., Uppington, G., Coordination of Management Activities - Mapping Organisational Structure to the Decision Structure, in Coordination Technology for Collaborative Applications, Organisations, Processes and Agents, in Conen, W. and Neumann, G. (eds.), Springer Verlag, 1998, pp.25-38.

[3]. Fox, M.S., Barbuceanu, M., Gruninger, M., An Organisation Ontology for Enterprise Modelling: Preliminary Concepts for Linking Structure and Behaviour. Computers in Industry, 29:123-134, 1996.

[4]. Fox, M.S., Chionglo, J. F., and Fadel, F. G., A Common Sense Model of the Enterprise, Proc. of the 2nd Industrial Engineering Research Conference, Norcross GA: Institute for Industrial Engineers, 1993, pp. 425-429.

[5]. Gruninger, M., Atefi, K., Fox, M.S., Ontologies to Support Process Integration in Enterprise Engineering, Computational and Mathematical Organization Theory, Vol. 6, No. 4, 2000, pp. 381-394.

[6]. Gruninger, M., Fox, M.S., Methodology for Design and Evaluation of Ontologies, Workshop on Basic Ontological Issues in Knowledge Sharing, IJCAI-95, Montreal.

[7]. Gruninger, M., Fox, M.S., The Role of Competency Questions in Enterprise Engineering", Proc. of the IFIP WG5.7 Workshop on Benchmarking, Trondheim, Norway, 1994.

[8]. Jennings, N. R., Coordination Techniques for Distributed Artificial Intelligence, in: G. M. P. O'Hare, N. R. Jennings (eds.). Foundations of Distributed Artificial Intelligence, John Wiley \& Sons, 1996, pp. 187-210.

[9]. Krogstie, J., Jørgensen, H. D., Quality of Interactive Models, Proc. of the 1st Int. Workshop on Conceptual Modelling Quality (IWCMQ’02), Tampere, Finland, Oct. 2002.

[10]. Mylopoulos, J., Characterising Information Modelling Techniques, in Handbook on Architectures of Information Systems, P. Bernus, K. Mertins, G. Schmidt (Eds.), Springer Verlag, 1998, pp. 17-58.

[11]. Petersen, S. A, Applicability of an Agent-based Approach for Supporting Virtual Enterprise Formation: A Case Study, IEEE 12 ${ }^{\text {th }}$ Intl. Workshops on Enabling Technologies: Infrastructure for Collaborative Enterprises (WETICE 2003), Linz, Austria, June 2003.

[12]. Petersen, S. A., Gruninger, M., An Agent-based Model to Support the Formation of Virtual Enterprises, Int. ICSC Symposium on Mobile Agents and Multi-agents in Virtual Organisations and E-Commerce (MAMA'2000), in Woolongong, Australia, 11-13 Dec. 2000. 
[13]. Petersen, S. A., Matskin, M., Agent Interaction Protocols for the Selection of Partners for Virtual Enterprises, Multi-agent Systems and Applications III, 3rd Int. Central and Eastern European Conference on Multi-Agent Systems, CEEMAS 2003, Prague, Czech Republic, June 2003. V.Marik, J. Muller, M. Pechoucek (Eds.), LNAI 2691, Springer-Verlag, 2003.

[14]. Vernadat, F. B., Enterprise Modelling and Integration Principles and Applications. Chapman and Hall Publishers, 1996.

[15]. Vesterager, J., Tølle. M., Pedersen, J. D., Globeman 21 Concepts, IMS1999, $2^{\text {nd }}$ International Workshop, Leuven, Belgium, Sept. 1999.

[16]. Wooldridge, M., Fisher, M., Huget, M. and Parsons, S., Model Checking Multi-agent Systems with MABLE, Proc. of the 1st Int. Joint Conference on Autonomous Agents and Multi-agent Systems, Bologne, Italy, July 2002.

[17]. Yin, R. K., Case Study Research Design and Methods, Second Edition, Sage Publications, 1994. 